\title{
165. THE PHYSIOLOGICAL ACTION OF ABNORMALLY HIGH TEMPERATURES ON POIKILOTHERMIC ANIMALS
}

\section{SOME CHANGES OCCURRING IN THE PHOSPHORUS - DISTRIBUTION OF THE HAEMOLYMPH OF INSECTS UNDER THE INFLUENCE OF ABNORMALLY HIGH TEMPERATURE}

\author{
By H. S. HOPF \\ From the Departments of Zoology and Applied Entomology and of \\ Biochemistry, Imperial College, London, S.W. 7
}

(Received 24 September 1940)

IN the first paper of this series [Fraenkel \& Hopf, 1940] it has been shown that the degree of saturation of the phosphatides of fully grown larvae of Calliphora erythrocephala Meig. and Phormia terra-novae R.D. is dependent on the temperature at which these larvae have been bred, irrespective of species. This means that these blowfly larvae will give the same iodine value for the fatty acids of their phosphatides for the same temperature of breeding, no matter to which of the two closely allied species they belong. The heat adaptation of these larvae was also tested and it was found that the larvae bred at higher temperatures were more resistant to heat treatment than larvae bred at lower temperatures. These results were discussed in the light of the lipoid liberation theory formulated by Heilbrunn [1924] and by Belehradek [1931] which attributes the cause of death from exposure to abnormally high temperatures to a "liberation" of the lipoids and explains the heat adaptation by the raising of the melting point in the more saturated fats of the larvae bred at high temperatures. It was pointed out by Fraenkel \& Hopf [1940] that, though this theory seemed to be supported by the correlation between the temperature of breeding and the degree of unsaturation in the animals in question, it left unexplained the phenomenon that the two species, while showing the same degree of unsaturation at the same temperature of breeding, had a vastly different resistance to high temperatures. Heat resistance therefore cannot depend solely on the nature of the lipoids concerned and investigations extending over a wider field were clearly desirable. The present communication records the results of the changes occurring in the haemolymph (body fluid) under the influence of abnormally high temperatures.

The reasons for choosing the haemolymph as material for anadysis are as follows. The haemolymph is the only body fluid in insects. If metabolic processes are involved in heat injury it is probable that some evidence of the changes will be found in the blood. Furthermore, the haemolymph is obtained with comparative ease from the body and is present in large amounts in the larvae of Calliphora erythrocephala and Phormia terra-novae, so that its analysis can be conveniently carried out. The need for a survey of the fate of the $P$ distribution during heat injury was shown by the observation (to be described 
below) that the total $\mathbf{P}$ content of the blood seemed to depend on the temperature at which the larvae were kept previous to the extraction of the haemolymph.

\section{Heat treatment of the larvae and preparation of the haemolymph for analysis}

Before obtaining the blood for analysis it was important that the insects should be in a reproducible condition of heat injury and that strictly standard conditions should be observed throughout. It was not thought advisable to use the larvae in a state in which they had actually been killed by heat, as postmortem changes were then likely to have occurred which had no direct connexion with the heat treatment given. It was therefore found best to use the larvae while they were still alive, though often in a state of heat rigor in which the high temperature had already produced irreversible injuries, so that normal flies would not develop from larvae thus treated.

Before death takes place in heat-treated larvae they develop heat rigor, a state in which they are motionless and fully extended, but will still react ("twitch") when some outside stimulus, such as pressing between the fingers, is applied. Unfortunately this state is not equally reversible in the two species, for while normal flies will seldom develop from Calliphora larvae after heat rigor, recovery is general in those of Phormia if the rigor has not lasted too long. Table 1 shows the differences between the two species. In these experiments

Table 1. Tests of survival from heat rigor

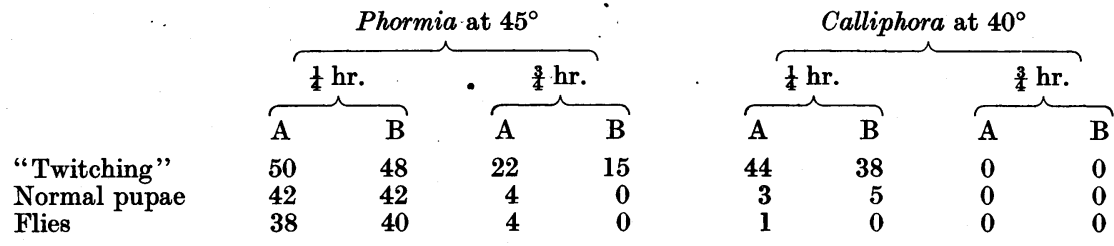

a temperature was chosen which was known to kill insects exposed to it within $5 \mathrm{hr}$., namely $45^{\circ}$ for Phormia and $39^{\circ}$ for Calliphora [Fraenkel \& Hopf, 1940]. In each case two batches of 50 larvae were used: one was restored to normal condition when 25 of the larvae had been motionless for $15 \mathrm{~min}$. and the other after $45 \mathrm{~min}$. further exposure to heat. A record was then taken of the number of larvae showing "twitching" $1 \mathrm{hr}$. after return to normal conditions, of the number of normal pupae formed and of the number of flies emerging from them. The experiments were carried out in duplicate, a different brood being used each time. The larvae were reared at $27^{\circ}$ and used fully grown.

It was decided that in the following experiments the Phormia larvae should be subjected to heat treatment until they passed into full heat rigor, while those of Calliphora should be given treatment sufficient only to produce the first evidences of this state. Both the temperature and time factors were accurately controlled.

The method of heat treatment has been fully described in the previous paper. After treatment the larvae were removed from the bottles, washed with distilled water, dried by gently rolling them between two sheets of filter paper and the body fluid was extracted in the following way.

A mixture of 9 parts of paraffin (Shell 24210) and 1 part of toluene was rendered air-free by allowing it to stand in vacuo for several hours until no more bubbles appeared. This fluid was neutral and was used to keep the blood ex- 
tracted from the larvae free from contact with air, dust or bacteria. A $10 \mathrm{ml}$. conical centrifuge tube was filled to the brim with this mixture and the tube placed upright in a conical flask, which served as a receptacle for the overflow when the blood was added.

Each larva in turn was taken up separately by the hind end and brought near the surface of the liquid. By pressing the hind end between the index finger and the thumb of the left hand all the blood collected in the front end of the animal. On puncturing the frónt end with a small sterilized scalpel or needle, a drop of blood issued from the wound, which was brought into contaet with the surface of the paraffin-toluene mixture, whereupon the drop sank to the bottom of the tube. The blood slowly accumulated there, causing an overflow of the protecting liquid. As it was essential to have the haemolymph uncontaminated by the gut of the larva, the incision was made at the mesothoracic segment in a slightly tangential direction.

In order to obtain $1 \mathrm{ml}$. of haemolymph, 90-110 larvae had to be treated in this way, heat-treated insects giving slightly less blood than the untreated controls.

After sufficient material had been collected the tubes were spun on a centrifuge for a few minutes. The blood corpuscles then formed a deposit and a layer of fat globules and fragments of body fat was found floating on top of the haemolymph proper. The latter appeared as an almost clear pale yellow fluid, usually somewhat darker when derived from heat-treated larvae than from the controls. The relative amounts of the three above-mentioned layers was approximately: serum $96 \%$, corpuscles $3 \%$ and fat $1 \%$ in the untreated larvae, while the fat content in the "sub-killed", i.e. irreversibly injured but still living, larvae was often as much as $2 \%$. After the larvae were dead the fat content of the haemolymph rapidly increased, fat being one of the first tissues of the insect body to disintegrate.

A graduated pipette was then carefully inserted into the tube and the fat layer was gently pushed aside so that the serum could be drawn off and analysed without coming into contact with air. On standing in the centrifuge tube for several hours the uppermost layers of the blood, where it came into contact with the paraffin, slowly blackened. Whether this blackening, which is doubtless a tyrosinase reaction, is caused by oxygen from the air diffusing through the paraffin or by an inter-surface action from the junction of the haemolymph with the paraffin-toluene mixture has not been determined. The blood serum thus obtained was in one instance used for a determination of the specific gravity and dry weight, the results being given in Table 2.

$\begin{array}{cccc}\text { Table 2. Specific gravity and dry weight of blood serum } \\ & & 45^{\circ} \text { for } 4 \mathrm{hr} . & \text { Control } \\ \text { Phormia } & \text { Sp. gr. } & 1.029 & 1 \cdot 018 \\ & \text { Dry wt. } & 17 \cdot 25 \% & 16 \cdot 55 \% \\ & & 40^{\circ} \text { for } 4 \mathrm{hr} . & \text { Control } \\ \text { Calliphora } & \text { Sp. gr. } & 1.025 & 1 \cdot 021 \\ & \text { Dry wt. } & 17 \cdot 10 \% & 16 \cdot 23 \%\end{array}$

\section{Phosphorus distribution in the haemolymph of heat-treated and of normal larvae}

If the total $P$ in the blood of heat-treated larvae is compared with the value for unheated controls, it is found that a considerable increase has taken place during the exposure to high temperatures (see Table 3). During the present 
work a batch of untreated larvae was in one instance kept in a refrigerator for 2 days as it could not be worked up at once and it was desired to arrest larval development and prevent pupation. The blood $\mathbf{P}$ of this particular batch was even lower than that of larvae treated at the normal temperature. The experiment was then repeated with aliquots of larvae from one brood, one sample being heat-treated, one exposed to cold and the last kept at $27^{\circ}$ - the temperature of breeding - and it was found that these conditions produced different total $\mathbf{P}$ contents in the haemolymph. That the processes involved were really connected with life functions was proved by the fact that the value for heatkilled larvae was the same before and after being kept in the refrigerator for a period of 2 days.

Table 3. The dependence of the total $P$ of blood on external temperature

\begin{tabular}{|c|c|c|c|}
\hline & \multirow[t]{2}{*}{ • } & \multicolumn{2}{|c|}{$\begin{array}{c}\text { Batches of larvae from the } \\
\text { same brood } \\
\text { mg. } P / \mathrm{ml} \text {. }\end{array}$} \\
\hline & & A & $\bar{B}$ \\
\hline Phormia & $\begin{array}{l}\text { Heat rigor at } 45^{\circ}\left(2 \frac{1}{2} \mathrm{hr} .\right) \\
\text { Normal } \\
\text { Kept at } 4^{\circ} \text { for } 2 \text { days }\end{array}$ & $\begin{array}{l}1 \cdot 08 \\
0 \cdot 74 \\
0 \cdot 68\end{array}$ & $\begin{array}{l}0.93 \\
0.69 \\
0.62\end{array}$ \\
\hline Calliphora & $\begin{array}{l}\text { Heat rigor at } 41^{\circ}\left(2 \frac{1}{2} \mathrm{hr} .\right) \\
\text { Normal } \\
\text { Kept at } 4^{\circ} \text { for } 2 \text { days }\end{array}$ & $\begin{array}{l}1 \cdot 22 \\
0 \cdot 91 \\
0 \cdot 85\end{array}$ & $\begin{array}{l}1 \cdot 30 \\
1 \cdot 04 \\
0 \cdot 92\end{array}$ \\
\hline
\end{tabular}

In view of the fact that $P$ plays an important part in such vital functions as carbohydrate metabolism and coenzyme activities a survey of the fate of various $\mathbf{P}$ compounds during heat treatment was undertaken. A combination of the methods applied by Needham \& Needham [1930] and by Needham et al. [1937] to embryological work was used in the following analyses, $\mathbf{P}$ being estimated throughout by the method of Martland \& Robison [1926].

The lipoid $\mathbf{P}$ (phosphatides) was determined by extraction of the haemolymph with alcohol and ether. For this purpose a measured quantity of blood, usually $2 \mathrm{ml}$., was mixed with analytical $\mathrm{P}$-free $\mathrm{CaSO}_{4}$ and allowed to dry over $\mathrm{H}_{2} \mathrm{SO}_{4}$ for 2-3 weeks. From the loss in weight during this operation the total blood solids were calculated. The dry mixture was then transferred to a Soxhlet thimble and extracted for 4 days with $97 \%$ alcohol, followed by ether (sp. gr. 0.720 ) for an equal time. The alcoholic extract was taken to dryness and the residue extracted 6 times with boiling ether. The $P$ in the combined ether extracts was regarded as the total lipoid $P$.

The residue in the Soxhlet thimble was then extracted 5 times in the cold for periods of $\frac{1}{2} \mathrm{hr}$. each with $1 \% \mathrm{HCl}$ and 5 times for similar periods with distilled water. Needham \& Needham [1930] combined this acid-soluble extract with the ether-insoluble residue from the alcohol extract, thus obtaining a total acidsoluble fraction in which they estimated the inorganic $P$, the pyrophosphate- $P$ and the stable organic $P$. Nevertheless, for the purposes of the present work it was found more expedient to use the method described by Needham et al. [1937], involving the use of trichloroacetic acid, for the acid-soluble fraction, since this gives a more detailed and physiologically more interesting partition of the P. Experience showed that the two methods give values for the total acidsoluble $\mathrm{P}$ which agree within a limit of error of $6 \%$. The $\mathrm{CaSO}_{4}$ in the thimble now retained only the protein compounds of $P$, and phosphoprotein- $P$ and nucleoprotein-P were estimated in the usual way.

Biochem. 1940, 34 
The acid-soluble $\mathrm{P}$ was fractionated as follows. $5 \mathrm{ml}$. of haemolymph from the same tube as that from which the material for the lipoid extraction was taken were precipitated with $25 \mathrm{ml}$. of ice-cold $5 \%$ trichloroacetic acid in a tube standing in a beaker with ice. The tube was allowed to stand in the refrigerator for $6 \mathrm{hr}$., after which the contents were filtered through an asbestos mat under pressure and the precipitate washed several times with small amounts of icecold trichloroacetic acid and water. The precipitate was rejected and the filtrate made up to $50 \mathrm{ml}$. The total acid-soluble $P$ was estimated in aliquots of $1 \mathrm{ml}$., each $\mathrm{ml}$. at this stage representing $0.1 \mathrm{ml}$. of haemolymph.

The remaining solution was then transferred to a centrifuge tube and fractionated according to the procedure of Needham et al. [1937], modified in some minor points to suit the material under investigation. The barium precipitate gave values for inorganic $P$, adenylpyrophosphate-P, hexosediphosphate-P and non-hydrolysing $P$, while the barium filtrate gave values for phosphagen-P and hexosemonophosphate-P; the last value in each group probably represented, in part, esters other than those of hexose.

Table 4. $P$ distribution in the haemolymph of heat-treated Calliphora larvae

\begin{tabular}{|c|c|c|c|c|c|c|c|c|c|}
\hline & 15 & ar. at 3 & $5^{\circ}$ & & ar. at 4 & & & Control & \\
\hline & A & B & $\mathrm{C}$ & A & $\mathrm{B}$ & $\mathrm{C}$ & $\mathrm{A}$ & B & C \\
\hline Total P & $1 \cdot 35$ & $1 \cdot 20$ & $1 \cdot 47$ & $1 \cdot 19$ & 1.02 & 1.08 & $1 \cdot 02$ & 0.92 & 0.98 \\
\hline Lipoid P & & $0 \cdot 1$ & $0 \cdot 14$ & & $0 \cdot 14$ & $0 \cdot 12$ & & 0.09 & $0 \cdot 10$ \\
\hline Total acid-soluble $\mathrm{P}$ & $1 \cdot 10$ & 0.98 & $1 \cdot 25$ & 0.95 & 0.82 & $0 \cdot 83$ & $0 \cdot 75$ & 0.71 & $0 \cdot 76$ \\
\hline anic $\mathbf{P}$ & $.0 \cdot 76$ & $0 \cdot 70$ & $0 \cdot 82$ & $0 \cdot 60$ & 0.52 & $0 \cdot 48$ & $0 \cdot 45$ & $0 \cdot 42$ & $0 \cdot 38$ \\
\hline lpyrophosphate-P & $0 \cdot 14$ & $0 \cdot 12$ & $0 \cdot 19$ & $0 \cdot 14$ & $0 \cdot 15$ & $0 \cdot 17$ & $0 \cdot 10$ & 0.08 & $0 \cdot 14$ \\
\hline "Hexosediphosphate-P" & $0 \cdot 04$ & 0.03 & $0 \cdot 07$ & $0 \cdot 04$ & $0 \cdot 02$ & $0 \cdot 06$ & 0.04 & 0.05 & $0 \cdot 07$ \\
\hline rolysing $\mathbf{P}$ & 0.05 & 0.04 & 0.08 & 0.05 & $0 \cdot 03$ & 0.08 & 0.05 & 0.06 & 0.07 \\
\hline & 0.02 & 0.02 & $0 \cdot 02$ & 0.04 & $0 \cdot 02$ & $0 \cdot 04$ & 0.04 & 0.05 & $0 \cdot 09$ \\
\hline semonophosphate-P & $0 \cdot 05$ & 0.04 & $0 \cdot 07$ & 0.05 & 0.04 & 0.02 & $0 \cdot 05$ & $0 \cdot 06$ & 0.01 \\
\hline Phosphoprotein- $\mathrm{P}$ & - & Trace & - & Trace & Trace & Trace & - & - & - \\
\hline Nucleoprotein-P & Trace & Trace & - & Trace & Trace & - & - & Trace & - \\
\hline
\end{tabular}

Table 5. P distribution in the haemolymph of heat-treated Phormia larvae; $m g . P . / m l$.

Total P

Lipoid $\mathbf{P}$

Total acid-soluble $P$

Inorganic $\mathbf{P}$

Adenylpyrophosphate-P

"Hexosediphosphate-P"

Non-hydrolysing $\mathbf{P}$

Phosphagen-P

Hexosemonophosphate-P

Phosphoprotein-P

Nucleoprotein-P
$15 \mathrm{hr}$. at $42 \cdot 5^{\circ}$

$\begin{array}{ccc}\mathrm{A} & \mathrm{B} & \mathrm{C} \\ 0.85 & 0.93 & 1.04 \\ 0.08 & 0.11 & 0.13 \\ 0.67 & 0.71 & 0.82 \\ 0.42 & 0.48 & 0.52 \\ 0.07 & 0.09 & 0.10 \\ 0.04 & 0.03 & 0.05 \\ 0.04 & 0.04 & 0.05 \\ 0.02 & 0.01 & 0.03 \\ 0.03 & - & 0.05\end{array}$

Trace Trace Trace

$\begin{array}{ccc}\overbrace{\text { A }} & \text { B } & \text { C } \\ 0.62 & 0.85 & 0.90 \\ 0.04 & 0.08 & 0.09 \\ 0.50 & 0.69 & 0.73 \\ 0.27 & 0.35 & 0.41 \\ 0.07 & 0.09 & 0.09 \\ 0.03 & 0.05 & 0.03 \\ 0.03 & 0.05 & 0.06 \\ 0.04 & 0.04 & 0.07 \\ 0.01 & 0.02 & 0.04 \\ - & \text { Trace } & - \\ - & \text { Trace } & \text { Trace }\end{array}$

In Tables 4 and 5 three sets of analyses are given for both Phormia and Calliphora larvae. They do not represent all the experiments carried out, but they constitute the only complete sets of results available and all the others were in accordance with those reproduced. In each case a brood of larvae was divided into 3 batches: one was heated to the "subkilled" state for $1 \mathrm{hr}$., the second to a temperature suitable to produce the same state within $15 \mathrm{hr}$., and the third kept as a control at the temperature of breeding $\left(27^{\circ}\right)$. Experiments have been carried out on larvae bred at higher and lower temperatures, and 
although the chemical analyses are not yet completed the results so far obtained do not show any divergence from those given with larvae bred at $27^{\circ}$ as long as the temperature employed produced the same state of heat injury in the same time of treatment as that used in the tabulated experiments.

In spite of all precautions to ensure the same state of beat injury in all cases, it was generally found that larvae treated for a long time at the lower temperature would survive the heat treatment for several hours longer than those treated at the high temperature. This may be attributed to the fact that at the high temperature the time increment between complete recovery and complete death is much smaller than at low temperatures, and that therefore the heat treatment was administered slightly on the excessive side to make sure that most of the insects were, in fact, effectively heat-injured.

In Tables 4 and 5 all numbers denote $\mathrm{mg}$. per $1 \mathrm{ml}$. of haemolymph and are calculated to the nearest $0.01 \mathrm{mg}$; A, B, C, refer to different broods of larvae. The results show for both species an increase in lipoid $\mathbf{P}$, inorganic $\mathbf{P}$ and adenylpyrophosphate-P after heat treatment. It will also be noticed that, with the exception of the adenylpyrophosphate- $P$, the increase is more pronounced in larvae treated at the lower temperature for the longer time.

\section{Discussion}

The above-mentioned results suggest that the $\mathrm{P}$ distribution in the haemolymph is dependent on the temperature to which the insect has been exposed before the blood is collected rather than on the temperature of breeding. The increase in $\mathbf{P}$ content after heat treatment of the larvae is not due merely to the increased concentration of the, blood, for the total $\mathrm{P}$ increased by $15-30 \%$ while the dry weight increased by only about $3 \%$.

It will be noted that there is a considerable increase in lipoid $\mathrm{P}$, representing on the average about a third of the total increase. This might suggest that fat has been melted or otherwise liberated from the body cells, thus providing a simple explanation of the heat injury. Against this view stands the fact that the increase in lipoid $\mathbf{P}$ is greater in larvae exposed for a long time to a low lethal temperature than in others treated for a shorter time at a higher temperature, though the heat treatment was, if anything, slightly more effective in the latter case.

A striking rise in inorganic $\mathbf{P}$ is evident, and the assumption that this is connected with the buffering systems of the haemolymph will be discussed in a later communication.

The method of fractional hydrolysis used to separate the esters precipitable with barium acetate is probably not ideal and there remains the possibility that other compounds besides those mentioned in the tables have been hydrolysed under the conditions employed. The figures quoted therefore have only a comparative value.

The adenylpyrophosphate-P has also risen quite considerably during heat injury and as adenylpyrophosphate is known to be a constituent of several enzyme systems it may be that heat injury produces a certain amount of enzyme activation in the haemolymph, a supposition which will be followed up in future work.

As has already been pointed out, the value recorded as hexosediphosphate-P represents only half of the total phosphate of this compound, for after $100 \mathrm{~min}$. hydrolysis only one of the two phosphate groups attached to the carbohydrate has been split off, leaving the more resistant hexosemonophosphate to be 
estimated as non-hydrolysing $P$. This should, of course, not be confused with the hexosemonophosphate originally present, which is not precipitated by barium acetate. No significant change appears to occur in the hexosediphosphate-P during heat injury. The amount of non-hydrolysing $P$ is slightly larger in all cases than that of the hexosediphosphate-P, which suggests the presence of other $\mathbf{P}$ compounds as well as hexosediphosphate. As regards the group of $\mathbf{P}$ compounds not precipitable with barium acetate, it is interesting that phosphagen is the only fraction which actually decreases during heat treatment. However, phosphagens are known to be very unstable and to hydrolyse easily, so that it is very probable that heat injury produces an actual breakdown of phosphagen in the blood. The nature of the phosphagen present in the larvae has not been investigated, but there is no evidence for an occurrence of creatine phosphates in animals of related phyla, while Baldwin \& Needham [1933] have shown that the phosphagen in the muscle of flies is arginine phosphate.

It is impossible to say whether the slight increase noticed in the hexosemonophosphate fraction in some of the cases examined represents a metabolic effect of heat treatment, especially as the small values impair the reliability of the results. In the author's opinion this increase, where it does occur, is more likely to be concerned with the incidental carbohydrate metabolism of the insects than with adaptation to high temperatare.

Comparing the two species it appears that the same tendency is observable in each case. The actual recorded changes for Calliphora are higher than those for Phormia but the relative increase is more pronounced in the latter. Thus the average increase in lipoid $P$ after long treatment is from about 0.1 to $0.15 \mathrm{mg}$. for Calliphora, and from $0 \cdot 06$ to $0 \cdot 11 \mathrm{mg}$. for Phormia, while the corresponding increases for acid-soluble $P$ are approximately from 0.75 to $1.10 \mathrm{mg}$. and from 0.5 to $0.75 \mathrm{mg}$. respectively.

Treatment for a short time at a high temperature produces less pronounced results than treatment for a long time at a somewhat lower temperature except in the case of the adenylpyrophosphate-P, which increased equally in both cases. This suggests that, if the larger increase at lower temperatures is to be attributed chiefly to the longer time of exposure, the adenylpyrophosphate (coenzymic) groups are probably liberated first from the cells, and that this process ceases on prolonged treatment.

Regarding the cause of the $\mathbf{P}$ increase as a whole, there appear to be two possibilities:

(a) the increase is due merely to a higher solubility at higher temperatures, accentuated by the greater permeability which heat-affected cells may possibly show, or

(b) this increase is connected with a series of metabolic processes instigated by the reaction of the organism to high temperatures, and therefore constituting an adaptation of the organism to the change in environmental conditions.

While the first possibility seems to explain satisfactorily the increase in inorganic $\mathbf{P}$ well enough, and might also perhaps explain the increase in coenzyme-P, the increase in phosphatide content is in strict contradiction to it. The total fat content of the haemolymph does not increase nearly as much as would account for the increase in phosphatide, were this product liberated in proportion to the other lipoids that occur in the body. We must therefore assume that there is a preferential release of phosphatide as compared with other fats. Furthermore, the phosphatides are known to form very stable complexes with proteins in the protoplasm, so that we may conclude that this particular increase is due to metabolic processes and not to a physically 
indiscriminate dissolving of tissue material. Similarly the apparently equal behaviours of inorganic and lipoid $P$ compounds make it possible that a connexion exists between the two. We may therefore assume that the changes described above do, in fact, represent an adaptation of the organism towards a change in its environmental conditions.

\section{SUMMARY}

Exposure of the larvae of Calliphora erythrocephala and Phormia terra-novae to abnormally high temperatures produces an increase in the lipoid $P$, the inorganic $P$ and the adenylpyrophosphate-P of the haemolymph. The function of the two latter groups might be regarded as being connected with buffering and coenzymic activities respectively. The increase is more pronounced for a long exposure to low lethal temperatures than for a short exposure to high lethal temperatures, except in the case of the adenylpyrophosphate-P which increases equally in both cases and is assumed to be liberated at an early stage.

The method of obtaining the blood and of standardizing the heat treatment is deseribed.

The author wishes to express his sincere gratitude to Prof. J. W. Munro, who originally suggested this work and made it possible for him to undertake it; to Prof. A. C, Chibnall for many valuable suggestions and for the hospitality of the Biochemistry Department; to Dr G. Fraenkel, under whose general supervision this work was carried out and to whose work on heat adaptation the present paper constitutes a sequel; and to Dr J. Needham and Dr H. W. Buston for much kindness and valuable advice. The costs of this investigation was partly defrayed by a grant from the Agricultural Research Council

\section{REFERENCES}

Baldwin \& Nèdham (1933). J. Physiol. 80, 221.

Belehradek (1931). Protoplasma, 12, 406:

Fraenkel \& Hopf (1940). Biochem. J. 34, 1085.

Heilbrunn (1924). Amer. J. Physiol. 69, 190.

Martland \& Robison (1926). Biochem. J. 20, 847.

Needham \& Needham (1930). J. exp. Biol. 7, 317.

—_ Nowinski, Dixon \& Cook (1937). Biochem. J. 31, 1185. 